\title{
Squamous cell carcinoma of the gallbladder
}

\author{
Fahmi Mghirbi, Mouna Ayadi, Wafa Karray, Yosra Yahyaoui, Khedija Meddeb, Amina Mokrani, Henda \\ Raies, Nesrine Chraeit, Amel Mezlini
}

Department of Medical Oncology, Salah Azaiez Institute, Tunis, Tunisia

Correspondence to: Mghirbi Fahmi. Department of Medical Oncology, Salah Azaiez Institute, Tunis, Tunisia. Email: mghirbifahmi@hotmail.fr.

\begin{abstract}
Gallbladder cancer is the most common malignant tumor of the biliary tract. The majority of cases are adenocarcinoma (AC). Pure squamous cell carcinoma (SCC) of gallbladder accounts only $3 \%$ of the malignant neoplasm of this organ. Many patients are at advanced stage when diagnosed and have bad therapeutic efficacy. At present, radical surgery is the only chance to gain long-term survival for patients with early-stage gallbladder cancers. Recent reports have shown a benefit of adjuvant chemoradiation in this type of tumor. At present, no therapy is defined for unresectable cancer of the gallbladder, especially for SCC.
\end{abstract}

Keywords: Carcinoma; gallblader neoplasm; squamous cell

Received: 11 September 2016; Accepted: 12 September 2016; Published: 20 October 2016.

doi: $10.21037 / \operatorname{tgh} .2016 .09 .07$

View this article at: http://dx.doi.org/10.21037/tgh.2016.09.07

\section{Case presentation}

A woman aged 67 years was admitted with a 2 months history of pain in the right hypochondrium and weight loss of $15 \mathrm{~kg}$ in 2 months. Physical examination revealed a palpable gallbladder. Abdominal ultrasonography showed heterogeneous gallbladder mass with echogenic center measuring $10 \mathrm{~cm}$ with invasion of the segment IV of the liver. The abdominal computed tomography (CT) revealed a solid cystic mass of the gallbladder bed measuring $7 \mathrm{~cm} \times 8 \mathrm{~cm} \times 8 \mathrm{~cm}$ with intratumoral calcification. This mass was extended to segments IV, V and VI of the liver and to the right colon. Intrahepatic bile duct was discreetly dilated. CT scan demonstrated also multiple liver metastases. Colonoscopy was performed and showed a protuberant lesion of the right colic angle which biopsy concluded metastasis of poorly differentiated squamous cell carcinoma (SCC) of the gallbladder. The tumor was judged unresectable. Then our patient was proposed for base supportive care regarding the poor performance status.

\section{Discussion}

Gallbladder carcinoma (GBC) is the commonest malignancy of the biliary tract. GBC shows wide geographic and ethnic variation across the globe. A recent review of the epidemiology of GBC reported highest incidence in Delhi $(21.5 / 100,000)$, followed by Karachi $(12.9 / 100,000)(1)$. Adenocarcinoma (AC) is the most common histological subtype of GBC followed by SCC and adenosquamous carcinoma (ASC). SCC of the GB represents only $2-10 \%$ of all GBC (2). GBSCC has been reported to have high proliferative rate and local invasiveness. Therefore patients with GBSCC are frequently diagnosed at an advanced stage with a bulky tumor and adjacent organ involvement (3). In our case, the tumor infiltrates the liver and the right colic angle. Charbit et al. reported that the growth rate of the squamous component (doubling time 81 days) was twice as fast as the adenocarcinomatous component (doubling time 166 days) (4). The exact mode of spread of these tumors is not clearly known. These tumors spread especially by direct local infiltration with a lesser incidence of metastases to the locoregional lymph nodes or distant organs. However, Chan et al. and Kim et al. reported high rate of lymph node metastasis in GBSCC $(5,6)$. Surgery is the corner stone of treatment especially when resection with wide margins is possible. However, the reported resectability rate of these tumors in different series was around 50\% $(5,6)$. Both Kim et al. and Chan et al. reported better survival in patients who underwent curative resection compared with patients who 
underwent $\mathrm{R} 1$ or $\mathrm{R} 2$ resection. For the locally advanced tumor extensive surgery should be considered, but no consensus regarding the appropriate extent of lymphadenectomy has been established. In our case the tumor was locally advanced and judged unresectable. Due to the relative rarity of the disease, the treatment of GBC based upon small case series and retrospective analysis and there are no consensus and randomized trial data concerning the benefit of adjuvant therapy. Horgan et al. conducted a systematic review and met analysis of published institutional and registry data to evaluate the benefit of adjuvant therapy for GBC. The authors reported a non-significant benefit of adjuvant therapy (7). When analyzed separately, outcome was found better in patients treated with chemoradiotherapy when compared with radiotherapy alone. The benefit was significantly higher for node positive and R1 resection. Some reports showed that external beam radiotherapy as an adjuvant treatment has some beneficial effect on survival (8).

\section{Conclusions}

GBSCC is a rare disease. Patients often presents with large, bulky tumors with involvement of adjacent organs. In spite of progress in surgical techniques and adjuvant chemotherapy, the prognosis of GBSCC remains poor.

\section{Acknowledgements}

This study is supported by Salah Azaiez Institute.

\section{Footnote}

Conflicts of Interest: The authors have no conflicts of interest

doi: $10.21037 / \operatorname{tgh} .2016 .09 .07$

Cite this article as: Mghirbi F, Ayadi M, Karray W, Yahyaoui Y, Meddeb K, Mokrani A, Raies H, Chraeit N, Mezlini A. Squamous cell carcinoma of the gallbladder. Transl Gastroenterol Hepatol 2016;1:78. to declare.

Informed Consent: Written informed consent was obtained from the patient for publication of this manuscript and any accompanying images.

\section{References}

1. Pandey M. Risk factors for gallbladder cancer: a reappraisal. Eur J Cancer Prev 2003;12:15-24.

2. Henson DE, Albores-Saavedra J, Corle D. Carcinoma of the gallbladder. Histologic types, stage of disease, grade, and survival rates. Cancer 1992;70:1493-7.

3. Kalayarasan R, Javed A, Sakhuja P, et al. Squamous variant of gallbladder cancer: is it different from adenocarcinoma? Am J Surg 2013;206:380-5.

4. Charbit A, Malaise EP, Tubiana M. Relation between the pathological nature and the growth rate of human tumors. Eur J Cancer 1971;7:307-15.

5. Chan KM, Yu MC, Lee WC, et al. Adenosquamous/ squamous cell carcinoma of the gallbladder. J Surg Oncol 2007;95:129-34.

6. Kim WS, Jang KT, Choi DW, et al. Clinicopathologic analysis of adenosquamous/squamous cell carcinoma of the gallbladder. J Surg Oncol 2011;103:239-42.

7. Horgan AM, Amir E, Walter T, et al. Adjuvant therapy in the treatment of biliary tract cancer: a systematic review and meta-analysis. J Clin Oncol 2012;30:1934-40.

8. Khan N, Afroz N, Haider N, et al. A case of pure endophytic squamous cell carcinoma of the gallbladder: a rare entity with aggressive behaviour. Turk Patoloji Derg 2012;28:181-3. 